\title{
Optimized design of robust resonator with distributed time-delay
}

\author{
Dan Pilbauer ${ }^{\mathrm{a}, \mathrm{b}}$ Tomáš Vyhlídal ${ }^{\mathrm{a}}$ Wim Michiels ${ }^{\mathrm{b}}$ \\ ${ }^{a}$ Dept. of Instrumentation and Control Eng., Faculty of Mechanical Engineering, \\ and Czech Institute of Informatics, Robotics and Cybernetics - CIIRC, Czech \\ Technical University in Prague, Technická 4, 16607 Praha 6, Czech Republic \\ ${ }^{\mathrm{b}}$ Department of Computer Science, KU Leuven, Celestijnenlaan 200A, B-3001 \\ Heverlee, Belgium
}

\begin{abstract}
An extension of the well-known delayed resonator concept is proposed with the objective to enhance its robustness in vibration suppression under guaranteeing stability of the overall set up. The novel resonator feedback is based on an acceleration measurements passing through a delay of polynomial distribution. The feedback design is formulated as an optimization problem over the parameter set formed by the polynomial coefficients and the gain of the delay free part. The first objective is to minimize the sensitivity of the resonator performance with respect to variations of the excitation frequency, i.e. to widen its frequency stop-band centered at the nominal excitation frequency. The second, equally significant objective is to guarantee that this does not lead to instability of the overall mechanical system coupled with the absorber and to ensure sufficient stability margin. The arising non-convex and non-smooth optimization problem is widely discussed and a penalty method is developed, where the unconstrained problem in every iteration step is solved using available software tools for optimization and spectral design of time delay systems. The proposed design technique is validated by simulations for two case study examples.
\end{abstract}

Key words: distributed time-delay, active vibration control, robust control, acceleration feedback, spectral method, delayed resonator

\section{Introduction}

In the task of vibration suppression of mechanical systems, both passive and active absorbers are among the fundamental tools. The positive impact of passive absorbers to vibration amplitude reduction has been known for 


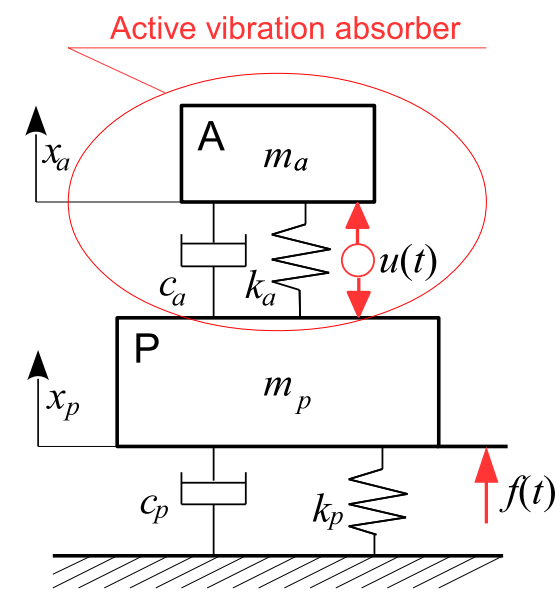

Fig. 1. Primary Structure $(\mathrm{P})$, with an active vibration absorber $(\mathrm{A})$ to suppress displacement $x_{p}$ induced by harmonic disturbance force $f(t)$

decades, see e.g. [1], [2], [3]. The main benefits of this passive approach is the inherent stability of the overall set-up and none energy needs to achieve the amplitude decay of the vibration. On the other hand, the frequency band where the passive absorber suppresses the vibration efficiently is relatively narrow, being centered at the natural frequency of the absorber. Besides, due to the fact that the absorber is never ideal, the vibration is not damped entirely even if the vibration frequency is identical with the natural frequency of the absorber. In order to extend applicable range of frequencies where the absorber is effective, methods for tuning the mechanical parameters of the absorber, particularly the stiffness of the dampers [1], [3], have been developed, see also [4] for broad-band damping tuned mass-damper design.

In this paper, we consider that the passive absorber is supplemented by an active feedback with the objective to improve the quality of vibration suppression. In Fig. 1, the overall set-up is depicted. It consists of an active vibration absorber (A), which is attached to the single degree-of-freedom primary structure $(\mathrm{P})$. By properly tuned feedback, force $u(t)$ can eliminate disturbing effects of harmonic oscillations $f(t)$ acting on the primary structure. This concept has been proposed in 1990s by N. Olgac and his co-workers, who introduced the delayed resonator (DR) control scheme [5], [6]. The method utilizes time-delay based feedback to modify the oscillatory frequency of the absorber resulting in entire suppression of undesirable oscillations. The absorber is tuned to an ideal resonator, marginally stable, where the DR acts as a perfect resonator and absorbs the vibration entirely. An advantage of using delayed resonator feedback lies in the simplicity of the implementation and the easy tuning rules (for a detailed discussion we refer to [5], [6]). From a methodological point of view, the design of resonators has a common feature with the design of input shapers which are widely used for pre-compensation of oscillatory modes of flexible systems. Namely, it is that both are concerned rather with the location of zeros close to the frequency to be suppressed than 
the location of poles. In [7] a detailed comparison is made between delaybased input shapers and delay-free shapers, highlighting the advantages of the former.

Note that the control feedback of the DR can be implemented using position, velocity or acceleration measurements, depending on the type of sensor selected for a particular vibration control application at hand. In this paper, the acceleration feedback is considered as it allows closing the feedback directly from the accelerometer sensors, which are easy to apply and of relatively low cost. Subsequent modifications of the resonator concept include a single-mass dual frequency absorber [8] or relative position feedback absorber in [9]. Next a related approach using a torsional vibrational absorber was proposed in [10]. An auto-tuning algorithm enhancing robustness against uncertainties in the absorber parameters was presented in [11]. An algorithm for multi-degree of freedom (MDOF) mechanical structures with multiple delayed resonators is described in [12], where multiple absorbers are used to suppress different harmonics. Stability of the vibration absorber using acceleration and displacement feedback was derived in [13] utilizing the Nyquist criterion.

Recently, a complete dynamics analysis of a DR with acceleration feedback was performed in [14]. The analysis shows negative effect when lumped-delayed feedback is taken from acceleration measurement, which results in undesirable neutral time delay system dynamics for both the resonator and its coupling with the primary structure. In order to mitigate this undesirable effect, an alternative distributed delay resonator (DDR) was proposed and analyzed in [15] resulting in a retarded spectral properties, which are more convenient. The second benefit of this novel resonator scheme is that the distributed delay provides filtering of the measured noise. By applying the method of Cluster Treatment of Characteristic Roots [16], it was shown in [14] and [15] that for both the DR and DDR, their operable frequency range is limited. From below, it is limited by the stability boundary, while the delay implementation aspects limit the range from the above - due to the exponential decay of the delay length with respect to growing frequency. A methodology for further extension of the operable frequency range was recently proposed in [17]. It is based on extending the feedback by a delay free factor virtually modifying the mass of the absorber and thus its natural frequency. Besides, implementation aspects of the resulting, so-called extended delay resonators have been discussed in [17]. To complete the state of the art on the resonator concepts, let us point to a delay free alternative to the DR proposed in [18], which is based on PI acceleration feedback. In [17], this PI scheme is further discussed and compared with the delay based schemes.

A fundamental feature of the delayed resonator concept is that the parameters of the primary structure do not play a role in the resonator tuning. The purpose of the design is only to suppress the vibration, while stability aspects 
of the overall set-up are left aside. As discussed in [14], [15], in fact, the resonator may decrease the stability margin or even destabilize the overall set-up. Thus, the first objective of the presented work is to design such a resonator feedback which will not only suppresses the vibration, but will also keep the set-up safely stable. The second objective is to enhance the robustness of the resonator against mismatch between the design and true excitation frequencies. As it results from the frequency domain analysis performed in application sections of [15], [17], the entire vibration suppression takes place only if the resonant frequency adjusted by the resonator is equal to the true excitation frequency. The performance of the resonator decays considerably even for very small differences in these frequencies. In the classical DR concept, an easy to apply feedback parameter tuning mechanism is applied to the given excitation frequency. If the frequency is fixed and known, the classical DR performs well. However, if the frequency is not constant and varies close to its nominal value within a bounded region, the classical DR needs to be continuously re-tuned. Besides, if the varying frequency is not known apriori and needs to be detected by a signal processing method (e.g. FFT [19]), the current estimate of the frequency is likely to be deflected as it is based on processing signal over a fixed time period being considerably longer than the period of excitation frequency. In order to handle this sensitivity issue, the design objective is to widen the frequency stop-band of the resonator. This robustness requirement will form the objective function of the optimization problem for the resonator feedback design. The stability of the overall set-up will then be included as a constraint expressed in a negative spectral abscissa bounded away, from zero (for a stabilizing controller design method for time-delay systems based on minimizing the spectral abscissa we refer to [20]).

Due to the use of the delayed resonator, the overall control scheme fits within the framework of intentionally using delays for control. Indeed, even though time-delays in control loops are usually associated with degradation of robustness and performance, and even instability, there are situations where delays are beneficial and used as controller parameters (for an overview of delay effects on stability we refer to [21]). Recent work in this direction includes reference [22], where an active vibration control scheme for an offshore steel jacked platform equipped with an active mass damper is designed. This scheme, based on a sliding mode control using feedback from both current and delayed state variables, has shown its benefits in terms of robustness and performance compared to existing approaches (we refer to [23] for an overview of control methods for this application). Another recent development is reported in [24] and the references therein, where proportional-plus delayed feedback controllers are designed. Controllers parameters are tuned for assigning multiple eigenvalues, aiming at minimizing the spectral abscissa function and, hence, the exponential decay rate of solutions. Complementary to this work, which is frequency domain based, the authors of [25] present time-domain, Lyapunov based methods for vector second-order systems. 
The paper is organized as follows. In Section 2, the concept of vibration absorber with both lumped and distributed delay is explained. Section 3 introduces novel type of delayed resonator together with a definition of objectives of the design which results in the optimization problem. In Section 4 the numerical results with simulation examples are described. In Section 5 the conclusions are presented.

\section{Preliminaries}

The dynamic model of the absorber, depicted in Fig. 1, with the physical parameters $m_{a}, c_{a}, k_{a}$ denoting the mass, the damping and the stiffness coefficients, can be described by

$$
m_{a} \ddot{x}_{a}(t)+c_{a} \dot{x}_{a}(t)+k_{a} x_{a}(t)=u(t)
$$

with $x_{a}$ being the displacement of the absorber and $u$ a control input. The damping ratio and the natural frequency of the absorber are given by $\zeta=\frac{c_{a}}{2 \sqrt{m_{a} k_{a}}}$ and $\Omega=\sqrt{\frac{k_{a}}{m_{a}}}$, respectively. The objective of the absorber is to suppress vibration of the primary structure being excited by a harmonic force $f(t)$ at the frequency $\omega_{f}$.

The model of the absorber-primary coupling according to Fig. 1 is given by

$m_{a} \ddot{x}_{a}(t)+c_{a} \dot{x}_{a}(t)+k_{a} x_{a}(t)-c_{a} \dot{x}_{p}(t)-k_{a} x_{p}(t)=u(t)$,

$m_{p} \ddot{x}_{p}(t)+\left(c_{a}+c_{p}\right) \dot{x}_{p}(t)+\left(k_{a}+k_{p}\right) x_{p}(t)-c_{a} \dot{x}_{a}(t)-k_{a} x_{a}(t)=-u(t)+f(t)$,

where $x_{p}(t)$ is the position and $m_{p}, c_{p}, k_{p}$ are the mass, the damping and the stiffness parameters of the primary structure.

Consider the Laplace transforms of the variables $U(s)=\mathcal{L}\{u(t)\}, X_{a}(s)=$ $\mathcal{L}\left\{x_{a}(t)\right\}, X_{p}(s)=\mathcal{L}\left\{x_{p}(t)\right\}$ and $F(s)=\mathcal{L}\{f(t)\}$. In general, the absorber feedback can be considered in the form

$$
U(s)=P(s) X_{a}(s),
$$

where $P(s)$ is the resonator feedback transfer function.

For the overall set-up (2)-(3), the transfer function between the excitation force $f$ and the position of the platform with feedback (4) is given by

$$
G_{x_{p}, f}(s)=\frac{X_{p}(s)}{F(s)}=\frac{R(s)-P(s)}{(R(s)-P(s)) V(s)+(P(s)-Q(s)) Q(s)}
$$


with

$$
R(s)=m_{a} s^{2}+c_{a} \mathrm{~s}+k_{a}
$$

being the characteristic function of the absorber (1), and

$$
\begin{gathered}
V(s)=m_{p} s^{2}+\left(c_{a}+c_{p}\right) s+\left(k_{a}+k_{p}\right), \\
Q(s)=c_{a} s+k_{a} .
\end{gathered}
$$

If the transfer function $P(s)$ is parameterized so that the characteristic equation of the resonator composed of the absorber (1) and the feedback (4), given by

$$
M(s)=R(s)-P(s)=0,
$$

has a root couple $s_{1,2}= \pm j \omega_{f}$, composing a pole couple of the resonator, then

$$
G_{x_{p}, f}\left(j \omega_{f}\right)=0
$$

indicating that no vibration at the given frequency $\omega=\omega_{f}$ are transferred in this $f$ to $x_{p}$ channel and the vibration is ideally suppressed. This means that external harmonic excitation at the given frequency $\omega_{f}$ should not impart any response on the primary structure, as the complete vibratory energy gets transmitted to the resonator absorber. In short, the resonator acts like an ideal vibration absorber for the exact input frequency $\omega_{f}$.

This expectation makes sense only if the entire system, the primary structure and the absorber, is stable, i.e. all the roots of the following characteristic equation

$$
(R(s)-P(s)) V(s)+(P(s)-Q(s)) Q(s)=0
$$

need to be located in the left half of the complex plane. For example, in $[5,9,10,11,12,14,15]$ and $[26]$, the above described resonator design concept is applied to the resonators with delayed feedback and also in [18] to the resonator with PI acceleration feedback. However, as already mentioned, stability of the overall system has not been a part of the design procedure so far.

\subsection{Resonator with lumped and equally distributed delay}

In order to show a parallel with the classical delayed resonator and the resonator with distributed delay the basic idea is briefly recalled first. The classical DR absorber with lumped delay, introduced in [5], has feedback given by

$$
u(t)=g \ddot{x}_{a}(t-\tau),
$$

for which the feedback transfer function is given as $P(s)=g e^{-s \tau} s^{2}$. The feedback is simply tuned by two parameters $g$ and $\tau$. The parameters can be 
obtained analytically, simply by assigning the dominant root couple of the characteristic equation of the resonator (1) and (11)

$$
\left(m_{a}-g e^{-s \tau}\right) s^{2}+c_{a} s+k_{a}=0
$$

on the imaginary axis for the given input excitation frequency $\omega_{f}[5,27]$.

Alternatively, feedback with distributed time delay was proposed in $[15,17]$ in the form

$$
u(t)=\frac{g}{\tau} \int_{0}^{\tau} \ddot{x}_{a}(t-\theta) d \theta,
$$

with the delay distributed equally over the time interval $[-\tau 0]$, which provides $P(s)=g \frac{1-e^{-s \tau}}{\tau s} s^{2}$. Similarly to (11), the feedback is also tuned only by two parameters $g$ and $\tau$ which are obtained via assigning the double root $s_{1,2}=$ $\pm j \omega_{f}$ to the characteristic equation

$$
\left(m_{a}-g \frac{1-e^{-s \tau}}{\tau s}\right) s^{2}+c_{a} s+k_{a}=0
$$

of the resonator (1)-(13), called distributed delay resonator (DDR).

As shown, for classical resonator with lumped or distributed delay only two parameters are free to be assessed which means that placing the complex conjugate couple of roots removes two degrees of freedom given by the parameters and no further modifications are possible. Extension has been proposed in [17] by introducing a delay-free part, for the DR

$$
u(t)=h \ddot{x}_{a}(t)+g \ddot{x}_{a}(t-\tau),
$$

or for DDR

$$
u(t)=h \ddot{x}_{a}(t)+\frac{g}{\tau} \int_{0}^{\tau} \ddot{x}_{a}(t-\theta) d \theta .
$$

The delay-free acceleration feedback part can virtually modify the mass of the absorber from $m$ to $m-h$ and thus to extend its range of operational frequencies. Another possible extension would be adding more absorbers as to get more parameters, as done in [12], where multiple resonators are used to suppress multiple harmonics. However adding more absorbers is not within our scope. This work focuses on extending the distributed delay feedback by including additional degree of freedom in the parameter set, which will be utilized for enhancing the robustness and guaranteeing stability of the overall set-up. 


\section{Main result - Design of a robust delayed resonator}

The robust resonator with a distributed delay (RDR) is designed as a straightforward extension of the distributed delay resonator given by coupling (1) with the feedback (16). The additional degree of freedom in the parameter space is achieved by considering a more complex distribution of the delay. In this aspect, this work is motivated by work of the author's team presented at [28], [29], where such distributed delays of enhanced complexity were applied to the design of input-shapers, i.e. time delay filters with the purpose to precompensate oscillatory modes of mechanical systems. Analogously to [29] a delay with polynomial distribution is applied to obtain the RDR feedback in the form

$$
u(t)=h \ddot{x}_{a}(t)+\int_{0}^{\tau} g(\theta) \ddot{x}_{a}(t-\theta) d \theta,
$$

where

$$
g(\theta)=\sum_{i=0}^{N} a_{i} \theta^{i} e^{-\lambda \theta},
$$

and $\tau$ denotes the delay length. As in (16), the feedback includes a delay free part with the gain $h$ which provides an additional parameter for the resonator tuning. Note also that the polynomial kernel function of the delay distribution $\sum_{i=0}^{N} a_{i} \theta^{i}$ with the real coefficients $a_{i}, i=0 . . N$ is weighted by an exponential term $e^{-\lambda \theta}$ with a real coefficient satisfying $\lambda>0$. This term is included for reasons of implementation of the distributed delay, which will be addressed below. The transfer function from $x_{a}$ to $u$ is given as

$$
P(s)=h s^{2}+s^{2} \sum_{i=0}^{N} a_{i} g_{i}(s, \tau)
$$

where $g_{i}(s, \tau)=\int_{0}^{\tau} \theta^{i} e^{-\lambda \theta} d \theta$. Together with delay-free parameter $h$ we get $N+2$ parameters to be assessed. In is important to note that the RDR reduces to the extended DDR (16) for the special case $N=0$ and $\lambda=0$.

\subsection{Robustness definition}

Consider the magnitude $\left|G_{x_{p}, f}(j \omega)\right|$ of the transfer function (5) between the excitation force $f$ and the position of the primary $x_{p}$. From the vibration suppression point of view, the objective is to minimize $\left|G_{x_{p}, f}(j \omega)\right|$ in the vicinity of $\omega=\omega_{f}$. This problem can be turned to minimizing the normalized transmissibility function

$$
T(\omega)=\left|\frac{R(j \omega)-P(j \omega)}{Q(j \omega)}\right|
$$


in the vicinity of $\omega=\omega_{f}$, which is fully determined by the absorber parameters in $R(s)$ and $Q(s)$ defined by (6) and (7) and by the parameters of active feedback $P(s)$ to be designed. The denominator $Q(s)$ normalizes the function (21) in order to achieve $\lim _{\omega \rightarrow 0} T(\omega)=1$. Note that by coupling equation (2) with the resonator feedback, the transmissibility can be interpreted as $T(\omega)=\frac{1}{\left|G_{x_{a}, x_{p}}(j \omega)\right|}$, where $G_{x_{a}, x_{p}}(s)=\frac{X_{a}(s)}{X_{p}(s)}$.

If the classical delayed resonator (11) or resonator with distributed delay (13) is applied, then $T(\omega)$ goes to zero for the nominal frequency $\omega=\omega_{f}$. For the frequencies in the vicinity of $\omega_{f}$, this function tends to rapidly increase and therefore, if there is an uncertainty in the excitation frequency, the quality of the vibration absorption is likely to decay.

Hereby the primary design objective for the RDR where $P(s)$ is defined by (19) and

$$
T(\omega, \tau)=\left|\frac{m_{a}(j \omega)^{2}+c_{a} j \omega+k_{a}-h(j \omega)^{2}-(j \omega)^{2} \sum_{i=0}^{N} a_{i} g_{i}(\omega, \tau)}{c_{a} j \omega+k_{a}}\right|,
$$

is to reduce this influence. Thus, the objective will be to widen the frequency range on which the function (21) is zero, or close to zero, at least. Note that this objective is analogous to extending the robustness of input shapers, where this requirement is imposed on the residual vibration function of extra insensitive input shapers [30], see also the references therein and [28], [29].

Thus, for a given frequency range $I_{\omega}=\left[\omega_{\min }, \omega_{\max }\right]$ in the neighborhood of the nominal frequency $\omega_{f}$ of the exciting force, we want to minimize an average of the frequencies that pass through this bandwidth. By discretizing this interval using a grid of points $\omega_{k}$, equidistantly or with Chebysev Chebyshev points distribution, the robustness criterion is considered as an average value of $T(\bar{\omega}, \tau), \bar{\omega} \in I_{\omega}$, given by

$$
r(p, \tau)=\frac{1}{N_{\omega}} \sum_{k=1}^{N_{\omega}} T\left(\omega_{k}, \tau\right)^{2}
$$

where $p=\left[\begin{array}{llll}h & a_{0} & \ldots & a_{N}\end{array}\right]^{T}$ is the vector of parameters to be determined.

Function (21) can be expressed as

$$
T(\omega, \tau)=|l(\omega, \tau) p+b(\omega, \tau)|
$$

where

$$
\begin{aligned}
& l(\omega, \tau)=\left[\begin{array}{llll}
-1 & -g_{0}(j \omega, \tau) \quad \ldots \quad-g_{N}(j \omega, \tau)
\end{array}\right] \frac{(j \omega)^{2}}{c_{a} j \omega+k_{a}}, \\
& b(j \omega)=\frac{m_{a}(j \omega)^{2}+c_{a}(j \omega)+k_{a}}{c_{a} j \omega+k_{a}},
\end{aligned}
$$


and, subsequently

$$
T(\omega, \tau)^{2}=p^{T} l(\omega, \tau)^{*} l(\omega, \tau) p+2 \Re\{\bar{b}(j \omega) l(\omega, \tau)\}+|b(j \omega)|^{2}
$$

where $*$ and $\bar{b}$ denotes the complex conjugate transpose and a complex conjugate number, respectively. Thus, the robustness measure takes the form

$$
r(p, \tau)=p^{T} H(\tau) p+B p,
$$

where

$$
\begin{aligned}
& H=\frac{1}{N_{\omega}} \sum_{i=0}^{N_{\omega}} l\left(\omega_{i}, \tau\right)^{*} l\left(\omega_{i}, \tau\right), \\
& B=\frac{2}{N_{\omega}} \sum_{i=0}^{N_{\omega}} \Re\{\bar{b}(j \omega) l(\omega, \tau)\} .
\end{aligned}
$$

Note that function (26) is a quadratic form in parameters $p$ if the delay length $\tau$ is considered to be fixed.

\subsection{Implementation of the feedback with distributed delay}

Following a general approach to approximate distributed delay system (16) by a linear system proposed in [31], see also the references therein, and considering $\ddot{x}(t)$ as the measured output, i.e. $y(t)=\ddot{x}(t)$, the feedback (17) can be rewritten into the form

$$
u(t)=h y(t)+\int_{0}^{\tau} C_{s} e^{A_{s} \theta} B_{s} y(t-\theta) d \theta,
$$

where

and

$$
A_{s}=\left[\begin{array}{ccccc}
-\lambda & 1 & 0 & \cdots & 0 \\
& -\lambda & \ddots & \ddots & \vdots \\
\vdots & & \ddots & \ddots & 0 \\
& & & -\lambda & 1 \\
0 & & \cdots & & -\lambda
\end{array}\right] \in \mathbb{R}^{N_{p}+1 \times N_{p}+1}
$$

$$
B_{s}=\left[\begin{array}{llll}
0 ! a_{0} & 1 ! a_{1} & \ldots & N ! a_{N_{p}}
\end{array}\right]^{\top}, C_{s}=\left[\begin{array}{llll}
0 & \ldots & 0 & 1
\end{array}\right] .
$$

The corresponding transfer function can then also be expressed as

$$
G_{s}(s)=\frac{u(s)}{y(s)}=h+C_{s}\left(s I-A_{s}\right)^{-1}\left(I-e^{-\tau\left(s I-A_{s}\right)}\right) B_{s} .
$$

This suggest a realization and implementation by a dynamic system

$$
\left\{\begin{array}{l}
\dot{z}(t)=A_{s} z(t)+B_{s} y(t)-e^{\tau A_{s}} B_{s} y(t-\tau), \\
u(t)=C_{s} z(t)+h y(t)
\end{array}\right.
$$


Given a number $\lambda>0$ the matrix $A_{s}$ (29) is Hurwitz and system (32) is exponentially stable. Also notice that the expression (32) is still linear in the parameters $p$.

\subsection{Stability of the overall set-up}

The absorber and primary structure coupled together can be described as

$$
\left\{\begin{array}{l}
\dot{x}(t)=A x(t)+B_{1} u(t)+B_{2} f(t), \\
y(t)=C \dot{x}(t)=C A x(t)+C B_{1} u(t)+C B_{2} f(t),
\end{array}\right.
$$

where the output is the measured acceleration and derivatives of states are in the output equation. Harmonic force $f(t)$ corresponds to the exciting force acting on the primary structure, and matrices $A, B_{1}, B_{2}$ correspond to a linear model of a mechanical system (2)-(3) translated into state space representation with a state vector $x=\left[\begin{array}{llll}x_{a} & \dot{x}_{a} & x_{p} & \dot{x}_{p}\end{array}\right]^{T}$. Matrix $C$ defines the measured acceleration of the absorber $C=\left[\begin{array}{llll}0 & 1 & 0 & 0\end{array}\right]$.

The system (33) and the feedback (28) create together System of Delay Differential Algebraic Equations (DDAE)

$$
\left\{\begin{array}{l}
\dot{z}(t)=A_{s} z(t)+B_{s} y(t)-e^{-A_{s} \tau} B_{s} y(t-\tau) \\
\dot{x}(t)=A x(t)+B u(t) \\
u(t)=C_{s} z(t)+h y(t) \\
y(t)=C A x(t)+C B_{1} u(t)+C B_{2} f(t)
\end{array}\right.
$$

which can be rewritten in a compact form of DDAEs [32] as

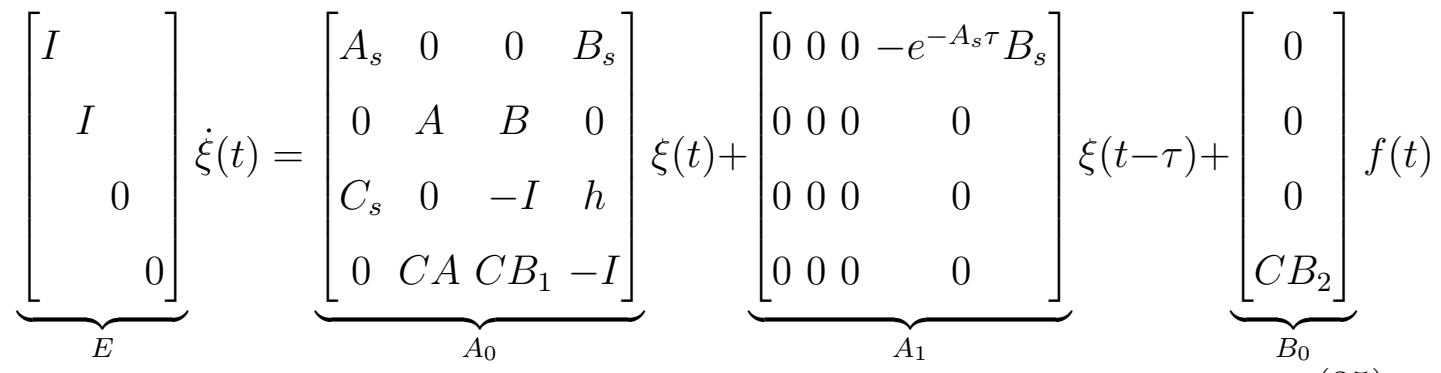

where $\xi=\left[\begin{array}{llll}z & x & u & y\end{array}\right]^{T}$ is a new state vector. With the new matrix notation the overall system can be written as

$$
E \dot{\xi}(t)=A_{0} \xi(t)+A_{1} \xi(t-\tau)+B_{0} f(t)
$$


For the resulting system, it is required that the spectrum of (36) lie safely in the left half of the complex plain. Defining the spectral abscissa of the system as

$$
\alpha(p):=\sup (\Re(s): s \in \Sigma(p)),
$$

with

$$
\Sigma(p):=\left\{s \in C: \operatorname{det}\left(s E-A_{0}(p)-A_{1}(p) e^{-s \tau}\right)=0\right\},
$$

the requirement on the stability is given as $\alpha<0$. Note that if $\alpha$ is very close to the imaginary axis, next to the undesirably small stability margin, it may result to undesirably long transients due to the low damping or slow modes brought by the rightmost roots. Therefore, it will be required so that the spectral abscissa is located sufficiently far to the left of the imaginary axis, i.e. $\alpha<\epsilon$, for some constant $\epsilon_{s}<0$.

\subsection{Robust resonator design as an optimization problem}

Recalling the objectives on the RDR, next to enhancing the robustness, the feedback (17) is to assure a sufficiently large stability margin of the closed loop of the absorber coupled with the primary (2)-(3) and the delayed feedback (17). This results in a design constraint on the spectral abscissa (37) given by

$$
\alpha(p)<\epsilon_{s}<0
$$

Thus the overall RDR design can be formulated as minimizing the objective function (21), translated into quadratic form (26), subject to the stability constraint (39). Thus, the RDR design can be expressed as an optimization task

$$
\min _{p} r(p, \tau), \quad \text { s.t. : } \quad \alpha(p)<\epsilon_{s}
$$

Due to the spectral inequality constraint, which is in general non-convex and non-smooth, the problem (40) is very difficult to solve [33]. We adopt a penalty method to handle the constraint: by modifying the objective function to

$$
f_{k}(p, \tau)=r(p, \tau)+\gamma_{k}\left\{\begin{array}{cc}
\alpha(s, p) & \alpha>\epsilon_{s} \\
0 & \alpha \leq \epsilon_{s}
\end{array}\right.
$$

where $\gamma_{k}>0$ is a penalty coefficient, the constrained optimization problem (40) can be turned to a series of unconstrained minimization problems

$$
\min _{p} f_{k}(p, \tau)
$$

The penalty coefficient $\gamma_{k}$ is increased (e.g. by a factor of 10) in each iteration $k$ until the solution is feasible. Thus, in each iteration the unconstrained problem is solved and the result is used as the initial guess for the next iteration. 
Once the solution of the unconstrained problem is feasible, it coincides with a solution of the original problem.

However, due to the presence of the spectral abscissa, the optimization problem cannot be handled by solvers for quadratic programming problems, because of the aforementioned non-smoothness and non-convexity of the spectral abscissa [34]. To tackle this difficult optimization task, a MATLAB program HANSO (Hybrid Algorithm for Nonsmooth Optimization) [35] is applied, which utilizes BFGS method using weak Wolfe line search, refined by the gradient sampling method [36]. The algorithm needs the evaluation of the objective function together with its derivatives with respect to the parameters, whenever the objective function is differentiable. These are obtained from the computation of the rightmost eigenvalues and associated eigenvectors, utilizing the Software for analysis and control of time-delay 1 by the team of W. Michiels [34].

The derivative of objective function (42) is defined as

$$
\frac{d f}{d p}=\frac{\partial r(p)}{\partial p}+\gamma\left\{\begin{array}{cl}
\frac{\partial \alpha(p, s(p))}{\partial p} & \alpha>\epsilon_{s} \\
0 & \alpha \leq \epsilon_{s}
\end{array}\right.
$$

(denoting $\frac{d f}{d p}$ a vector whose $i$-th component is given by the partial derivative of $f$ with respect to the $i$-th component of $p$, etc.), where the first part is simply

$$
\frac{\partial r(p)}{\partial p}=2 H p+B
$$

with $H$ and $B$ defined in (27) and the second part results in

$$
\frac{\partial \alpha}{\partial p}=\left\{\begin{array}{c}
\frac{\partial \alpha}{\partial h}=-\frac{u^{*} \frac{\partial M}{\partial h} w}{u^{*} \frac{\partial M}{\partial M} w} \\
\frac{\partial \alpha}{\partial a_{0}}=-\frac{u^{*} \frac{\partial M}{\partial \Omega_{0}} w}{u^{*} \frac{\partial M}{\partial s} w} \\
\vdots \\
\frac{\partial \alpha}{\partial a_{N}}=-\frac{u^{*} \frac{\partial M}{\partial a_{N}} w}{u^{*} \frac{\partial M}{\partial s} w}
\end{array}\right\}=\Re\left\{-\frac{1}{u^{*} \frac{\partial M}{\partial s} w}\left[\begin{array}{c}
u^{*} \frac{\partial M}{\partial h} w \\
u^{*} \frac{\partial M}{\partial a_{0}} w \\
\vdots \\
u^{*} \frac{\partial M}{\partial a_{N}} w
\end{array}\right]\right\}
$$

where $u$ and $w$ are the left and right eigenvector of the closed loop system, i.e.,

$$
\begin{gathered}
M(s, p) w=0, \\
u^{*} M(s, p)=0,
\end{gathered}
$$

with

$$
M(s, p):=\left(s E-A_{0}(p)-A_{1}(p) e^{-s \tau}\right) .
$$

$\overline{1}$ Available from: http://twr.cs.kuleuven.be/research/software/delay-control/ 


\subsubsection{Note on the selection of the constant $\lambda$ and time delay $\tau$}

In the proposed method a length of the delay $\tau$ is to be selected as a fixed value. The length of the time delay $\tau$ could be considered as additional design parameter, but it would further increase the complexity of the optimization problem and also the time needed to solve it. As the parameters of the system (2)-(3) and the range of exciting frequencies are known, a sufficiently good value of the delay can be estimated. The delay is suggested to be at least one period of the nominal excitation frequency, i.e.,

$$
\tau=c \frac{2 \pi}{\omega_{f}}, c \geq 1
$$

The choice of $\lambda$, once again, induces a trade-off. For large $\lambda$, the additional dynamics are very fast and do not affect the overall control system performance. However, since the kernel basis functions (18) have a rapid decay, it might become more difficult to find parameter values for the resonator satisfying the design requirements, affecting the performance. Therefore, in our implementation we select $\lambda$ as

$$
\lambda=\frac{\omega}{2 \pi}=\frac{1}{\tau}
$$

which gives a good compromise.

\subsection{Further comments on the implementation of the resonator feedback}

Concerning the implementation of the resonator feedback (17)-(18) on a physical control unit, the first option is to implement numerically the dynamic system (32). In a standard control system design tool (Matlab-Simulink, Labview) the implementation of (32) is straightforward, composed of an interconnection of several predefined functionalities/blocks (state space model, time delay, matrix gain, etc.). The numerical solution then involves a discrete approximation of a single time delay and application of a standard integration scheme, e.g. Runge-Kutta (if the sampling period $\Delta t$ is small, first order explicit Euler or second order Heun schemes should be sufficient).

An alternative option is to directly approximate the distributed delay in (17)-(18) leading to

$$
y(t) \approx h y(t)+\sum w_{k} g\left(\theta_{k}\right) u\left(t-\theta_{k}\right)
$$

where $\theta_{k}$ and $w_{k}$ are nodes and weights of the quadrature formula. This leads to an implementation of the distributed delay term as a standard FIR filter, 
which is relatively straightforward [19]. However, let us point to the fact that if the numerical integration method is not selected appropriately, this methods may lead to instability (see [37] and the references therein).

As regards the optimization procedure for adapting the parameters to a current value of the excitation frequency, it can be performed on an upper level of the control system. An alternative option is to tabularize the parameters in advanced by performing the optimization procedure over a given operable frequency range. Note that due to the enhanced robustness, the grid step of the discretized frequency range can be relatively large.

\section{Case study examples}

Consider the interconnection of the active absorber and primary structure as given by (2)-(3) and shown in Fig. 1 with parameters given as $m_{a}=$ $0.223 \mathrm{~kg}, c_{a}=1.273 \mathrm{kgs}^{-1}, k_{a}=350 \mathrm{Nm}^{-1}$ for the absorber and $m_{p}=1.52 \mathrm{~kg}, c_{p}=$ $10.11 \mathrm{kgs}^{-1}, k_{p}=1960 \mathrm{Nm}^{-1}$ for the primary structure, which were also considered in [15] and [17]. The natural frequency of the absorber at which it performs the best when the resonator feedback is inactive is given as $\omega_{n}=$ $\sqrt{\frac{k_{a}}{m_{a}}}=39.617 \mathrm{~s}^{-1}$. Next to the RDR feedback in the proposed form (17)-(18), the classical DR (11) with lumped delay is considered for comparison.

\subsection{Case 1 - stop-band extension centered at the natural frequency}

First, we assume that the primary structure is excited by a periodic force $f(t)=\Delta f \sin \left(\omega_{f} t\right)$ with amplitude $\Delta f=10 N$ and the nominal frequency $\omega_{f_{n}}=\omega_{n}=39.617 \mathrm{~s}^{-1}$. The parameters of the DR assigning a couple of poles $\pm j \omega_{f_{n}}$ are given as $g_{D R}=0.0321 \mathrm{~kg}$ and $\tau_{D R}=0.0396 \mathrm{~s}$. As can be seen, the gain is of relatively low value as only a slight adjustment of the absorber dynamics is needed to generate an ideal resonator.

For the RDR, the required low sensitivity frequency range was chosen as $I_{\omega}=\left[\begin{array}{ll}0.98 & 1.02\end{array}\right] \omega_{f_{n}}$, with $N_{\omega}=100$ points over the region by Chebysev point distribution. The stability margin has been selected as $\epsilon_{s}=-0.5$. Running the overall optimization procedure repeatedly, taking into consideration the complexity of the feedback and achieved quantitative characteristics, very good results have been achieved e.g. for $N=3$ and $c=8$ determining the delay length $\tau=1.2688 \mathrm{~s}$ and $\lambda=0.7882 \mathrm{~s}^{-1}$ by (48) and (49), respectively. The resulting parameter set is given by $p=[-0.0001,1.5758,0.5519,-1.0299,0.1815]^{T}$. The impulse response of the feedback transfer function $\frac{1}{s^{2}} P(s)(P(s)$ given by (19)) between the measured acceleration $y=\ddot{x}_{a}$ and the action force $u$ is 


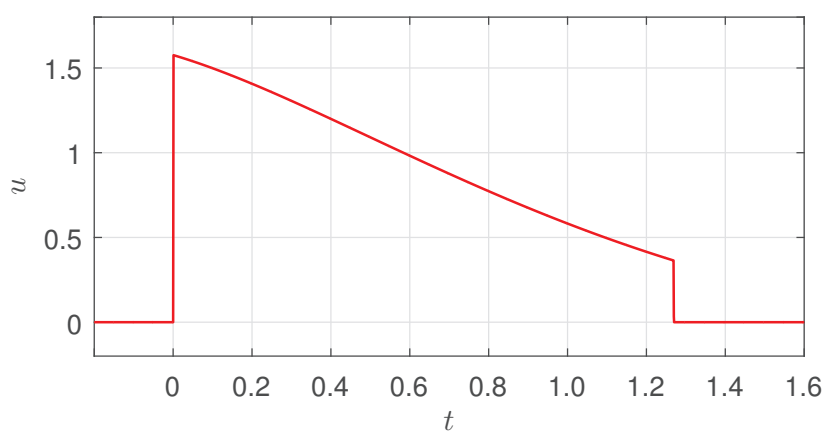

Fig. 2. Case 1 - Impulse response of the transfer function $\frac{1}{s^{2}} P(s), P(s)$ given by (19), between the measured acceleration $y=\ddot{x}_{a}$ and the action force $u$ of RDR.

given in Fig. 2. Notice that the parameter $h=-0.0001 \mathrm{~kg}$ virtually modifying the mass and thus the natural frequency of the absorber is close to zero. This is in agreement with the result presented in [17] for structurally simpler resonators, stating that if the system is excited by a periodic force on a natural frequency, no virtual adjustment of the absorber mass is needed. Thus, the impulse response is predominantly given by the distribution of the delay $g(\theta)$. Notice also that the delay length is considerably longer than the delay $\tau_{D R}$ of the DR. This can be considered as a positive feature, taking into account the implementation point of view and the fact that the weighted average performed by the distributed delay is taken over a considerably large region of time, which is beneficial for signal filtration.

The results achieved by the designed RDR feedback, in comparison with the results by DR feedback, are shown in Fig. 3-5. As can be seen in the
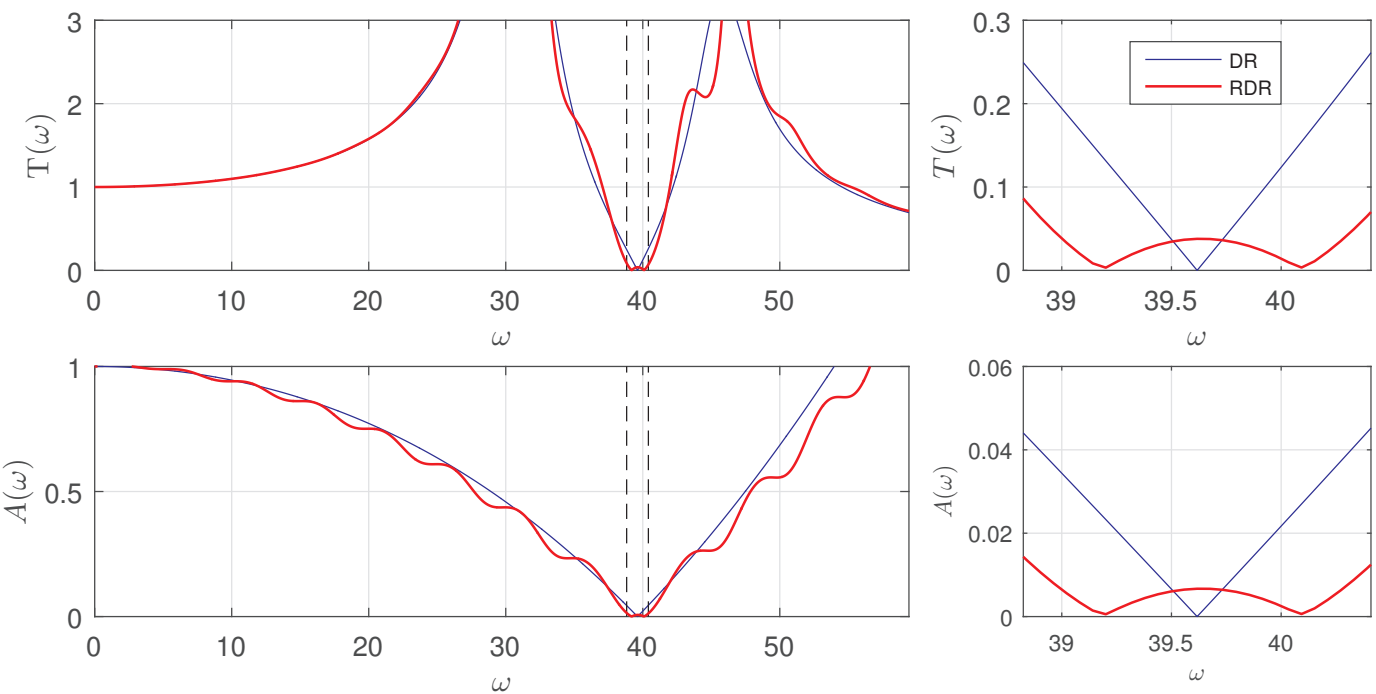

Fig. 3. Case 1 - Transmissibility characteristics: Top - Resonator RDR and DR with feedbacks (17)-(18) and (11), given by (21) determining the objective function. Bottom - Overall set-up $A(\omega)=\frac{\left|G_{x_{p} f}(j \omega)\right|}{\lim _{s \rightarrow 0} G_{x_{p} f}(s)}$ 


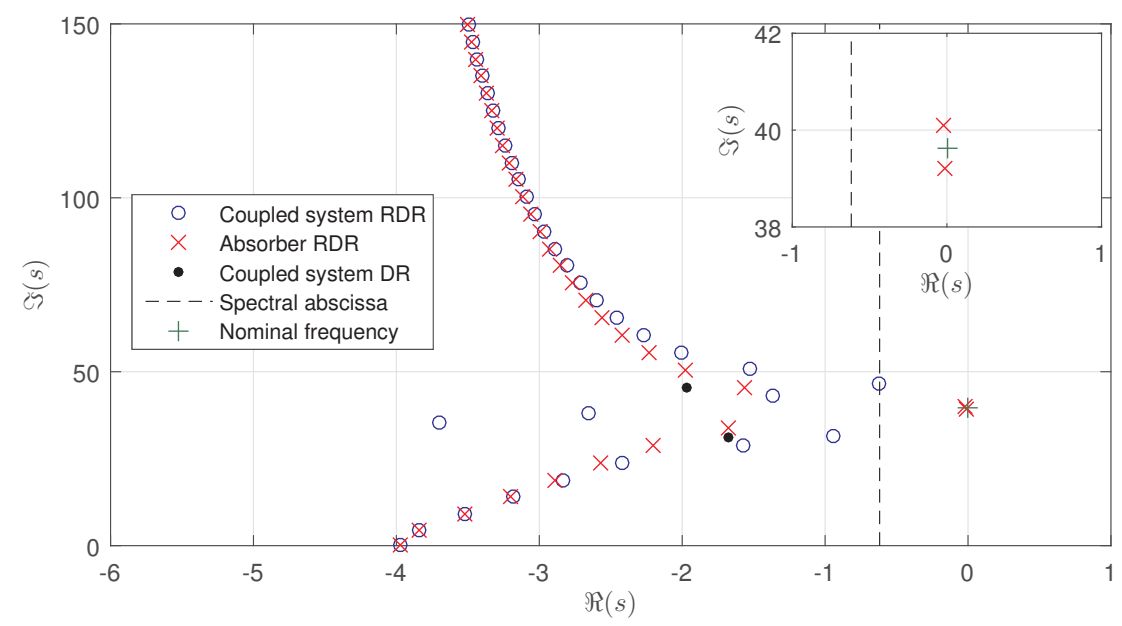

Fig. 4. Case 1 - Spectra of the RDR and its coupling with the primary structure; spectrum of DR coupling with the primary structure.

normalized transmissibility curves of the resonators in Fig. 3-top, the bottom of the frequency stop-band of RDR was extended (compared to the DR) to the desired interval $I_{\omega}$. Notice that this is at the cost of loosing ideal vibration suppression for the nominal frequency as $T\left(\omega_{f_{n}}\right)>0$ for the RDR (compared to $T\left(\omega_{f_{n}}\right)=0$ for DR). The properties of the resonators are well transferred to the transmissibility of the overall set-up $A(\omega)=\frac{\left|G_{x_{p} f}(j \omega)\right|}{\lim _{s \rightarrow 0} G_{x_{p} f}(s)}$ given in Fig. 3-bottom.

Concerning the spectral properties, it can be seen in Fig. 4 that the requirement on the spectral abscissa $\alpha<-0.5$ has been achieved. At the spectrum of the RDR, it can be seen that instead of one root assigned at the nominal frequency $j \omega_{f_{n}}$ (as it is for DR, the spectrum of which is not visualized), we have a couple of roots located nearby. These roots are responsible for the almost zero points of DDR characteristics in Fig. 3. Note that, from this point of view, both the spectral and transmissibility characteristics are analogous to the spectral and residual vibration characteristics of two-hump extra insensitive (EI) input shaper [30], see also references therein. Additional properties to notice are that i) the spectrum of the overall set-up tends to asymptotically follow the spectrum of the RDR - both being of retarded character, ii) also the spectrum of the set-up with DR is safely stable, in agreement with the analysis performed in [14].

Finally, the simulated performance of the RDR and DR in vibration suppression at the primary structure is compared in Fig. 5, where the resonator feedback is switched on at $t=2 \mathrm{~s}$. At excitation by the nominal frequency $\omega_{f_{n}}$ shown in the topmost subplot, in agreement with the transmissibility curves in Fig. 3, the DR suppresses vibration entirely, while small residual vibration can be observed for RDR. However, even a very slight change of the excitation frequency by $\pm 1 \%$ with respect to $\omega_{f_{n}}$, shown in the second and third 

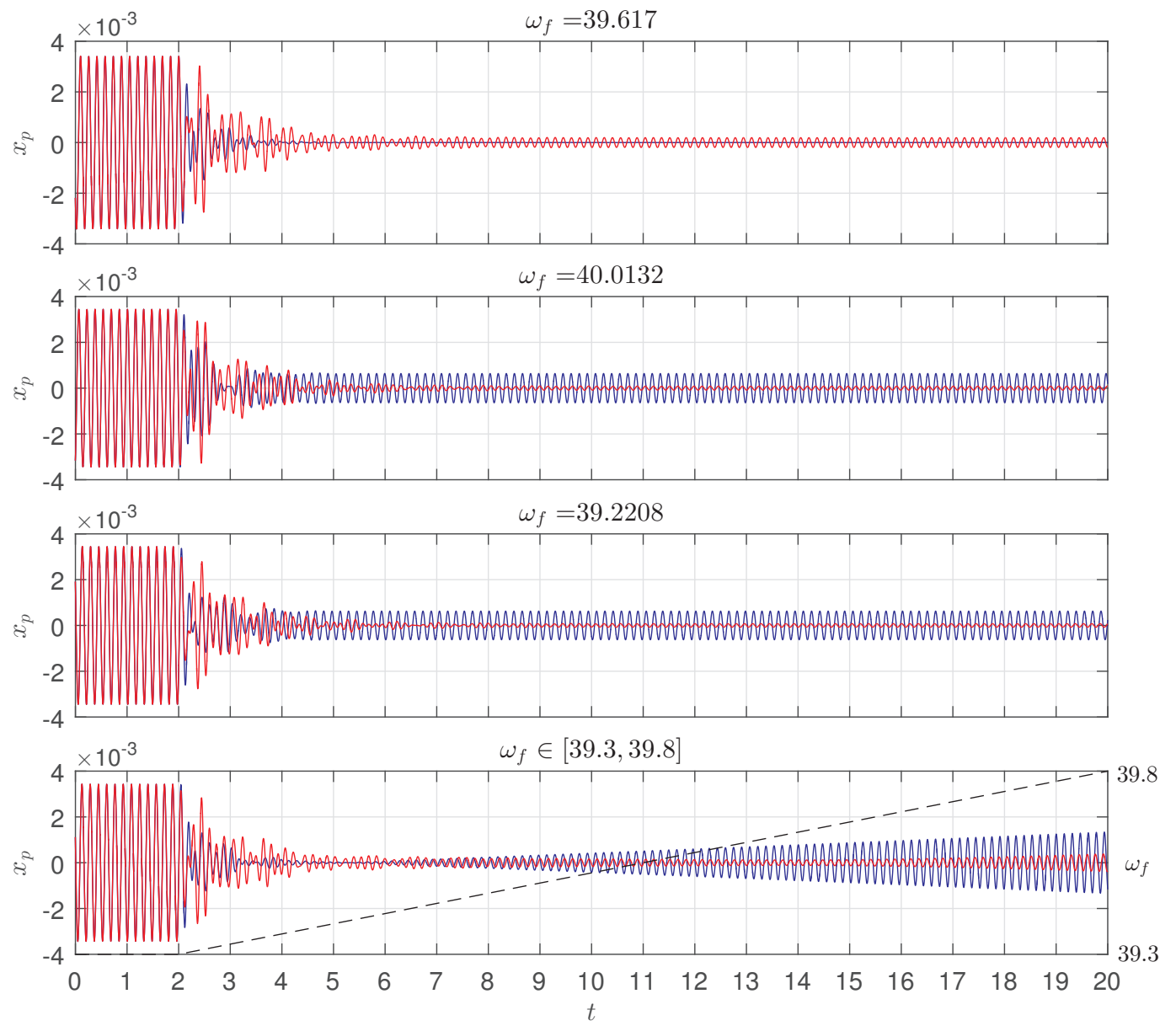

Fig. 5. Case 1 - Vibration suppression by DR (blue curve) and RDR (red curve) resonators, which are switched on at $t=2 \mathrm{~s}$ for various frequencies. The nominal case with $\omega_{f_{n}}=39.617 \mathrm{~s}^{-1}$ is at the topmost figure. In the downmost subplot the response to a chirp signal with slowly varying frequency (dashed line) in the interval $\omega_{f} \in[39.3,39.8]$ is demonstrated.

subplot from the top, causes considerable performance decay for the DR. It is not the case for the RDR where the residual vibration is even smaller than for the nominal case, also in agreement with Fig. 3. Additionally, in the downmost subplot of Fig. 5 we illustrate the behavior under changing the frequency from $\omega_{f}=39.3$ to $\omega_{f}=39.8$ following a chirp signal highlighted in the figure. As can be seen, considerably better vibration suppression is achieved by the RDR, assuming both the resonators remained tuned to the nominal excitation frequency $\omega_{f_{n}}$.

Notice that, in this case, even $1 \%$ difference between the true and nominal frequency caused considerable degradation of vibration suppression by the DR. Such a small difference is likely to appear in practical applications as the detection of the excitation frequency can be inaccurate due to various reasons, e.g. due to its slight variation, effect of the noise, sensor inaccuracy, etc. This 
is the case when the application of the proposed RDR would be reasonable. However, if the excitation frequency is known, the re-tuned DR would serve well, providing the entire vibration suppression.

Thus, to sum up, all the presented results confirm that both the RDR design goals have been achieved. The proposed RDR truly has enhanced robustness and ensures stability of the overall set-up.

\subsection{Case 2-wider stop-band and unstable set-up with the DR}

In the second case study example, we demonstrate that the RDR can be used for suppression of frequencies for which the classical DR destabilizes the overall set-up. Besides, a wider stop-band represented by $I_{\omega}=[0.951 .05] \omega_{f_{n}}$ is considered. Again, the primary structure is excited by $f(t)=\Delta f \sin \left(\omega_{f} t\right)$

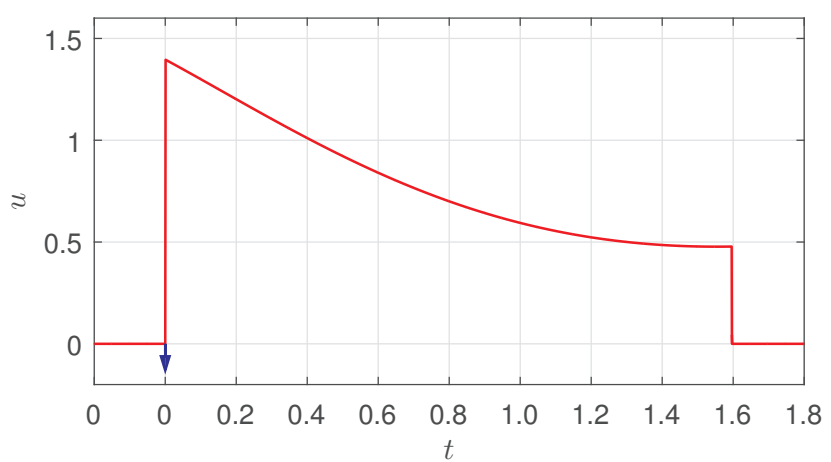

Fig. 6. Case 2 - Impulse response of the transfer function $\frac{1}{s^{2}} P(s), P(s)$ given by (19), between the measured acceleration $y=\ddot{x}_{a}$ and the action force $u$ of RDR.

with $\Delta f=10 N$. However, the nominal excitation frequency $\omega_{f_{n}}=31.5 \mathrm{~s}^{-1}$ is lower compared to the natural frequency of the absorber $\omega_{n}$. The parameters of the DR determined for the nominal frequency $\omega_{f_{n}}$ are given as $g_{D R}=0.1359 \mathrm{~kg}$ and $\tau_{D R}=0.0901 \mathrm{~s}$. Let us note that for the given excitation frequency and the given absorber with the damping $\zeta=0.072$, the DR itself is close to its (quasi)stability boundary. According to the stability maps derived in [14], for the given $\zeta$ the DR changes its posture from the quasi-stable to unstable resonator for $\omega_{f}<0.78 \omega_{n}$ (i.e. the stability switching frequency is $\omega_{f}=30.90$ ).

For the RDR, except the range $I_{\omega}$, all the optional parameters remain the same as in the Case 1, i.e. $N=3, c=8, N_{\omega}=100$, for which $\tau=1.5957 \mathrm{~s}$ and $\lambda=0.6267 \mathrm{~s}^{-1}$ result. A feasible solution minimizing the objective function, which includes the requirement on $\alpha<\epsilon_{s}, \epsilon_{s}=-0.5$ has been achieved providing the parameter set $p=\left[\begin{array}{llll}-0.1302 & 1.3958-0.0697-0.5853 & 0.3704\end{array}\right]^{T}$. The impulse response of the RDR feedback transfer function $\frac{1}{s^{2}} P(s)$ is given in Fig. 6. Notice that in this case, a considerably higher magnitude of the delay free parameter $h=-0.1302 \mathrm{~kg}$ (indicated as a weighted Dirac impulse 

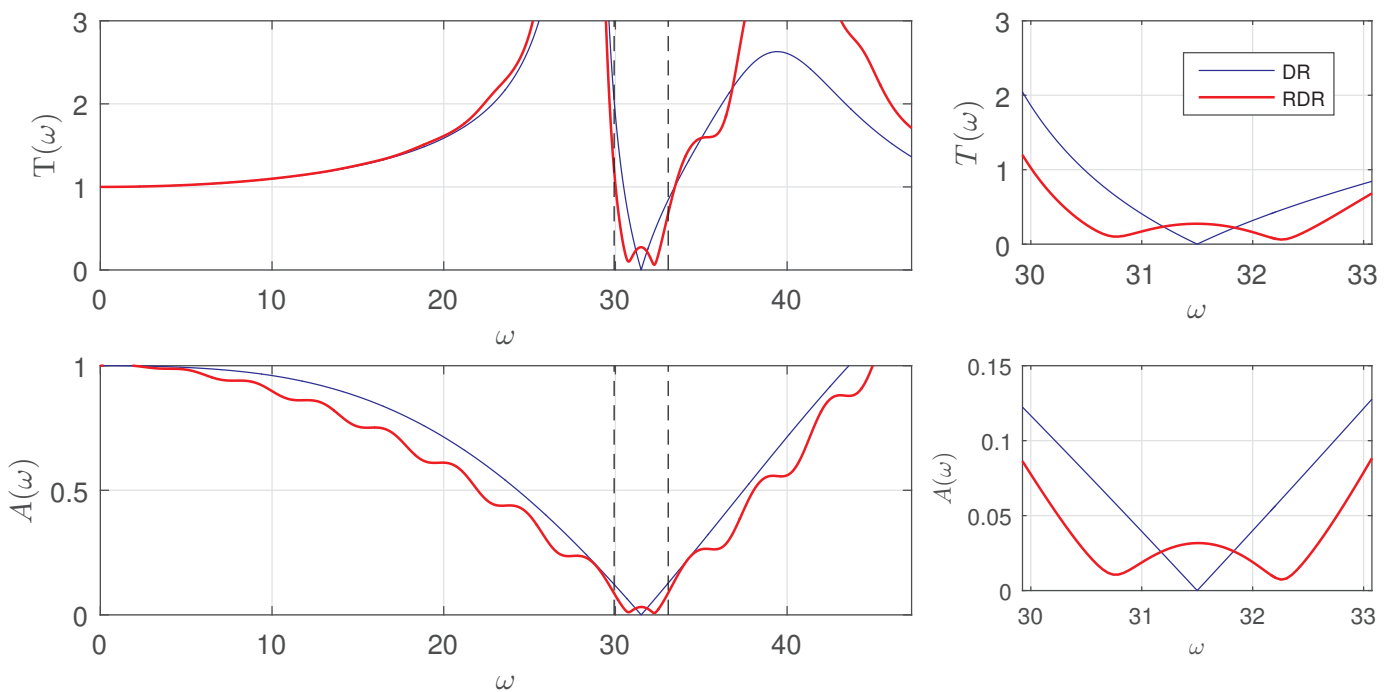

Fig. 7. Case 2 - Transmissibility characteristics: Top - Resonator RDR and DR with feedbacks (17)-(18) and (11), given by (21) determining the objective function. Bottom - Overall set-up

in Fig. 6) has been achieved which virtually increases the mass of the absorber from $m_{a}=0.225 \mathrm{~kg}$ to $\bar{m}_{a}=0.0928 \mathrm{~kg}$. This will practically shift the natural frequency to $\omega_{n}=\sqrt{\frac{k_{a}}{\bar{m}_{a}}}=31.48 \mathrm{~s}^{-1}$, which is very close to the excitation frequency $\omega_{f_{n}}$. This result is in agreement with the analysis performed in [17], where the key finding is that the delay free part should be used to modify virtually the absorber mass so that the natural frequency of the absorber is equalized with the excitation frequency.

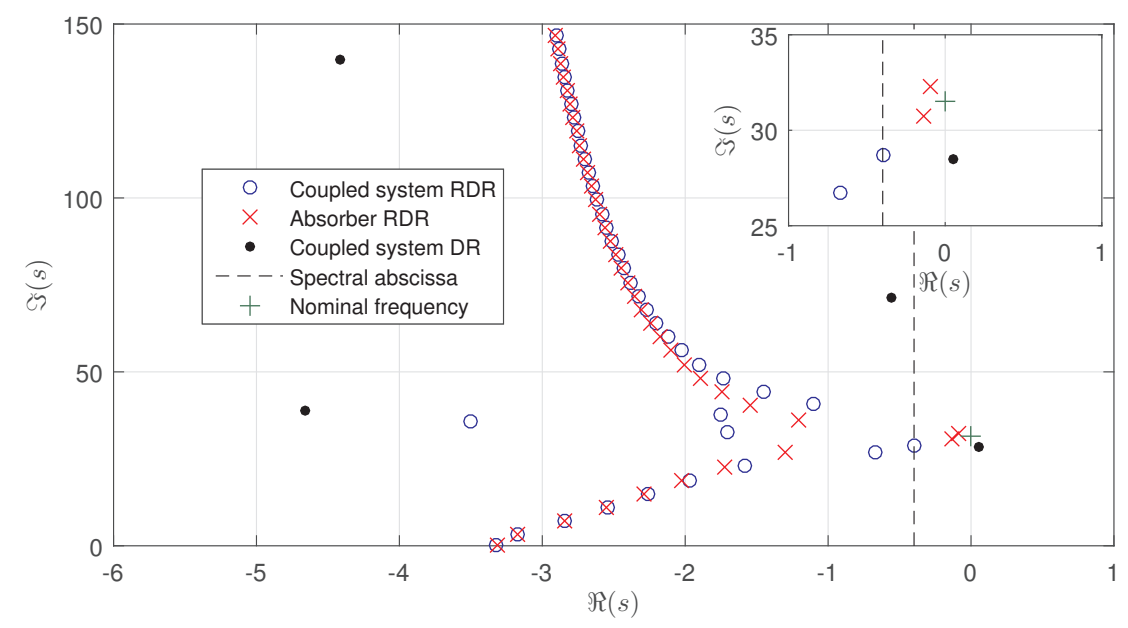

Fig. 8. Case 2 - Spectra of the RDR and its coupling with the primary structure; spectrum of DR coupling with the primary structure.

The results for Case 2 are shown in Figs. 7-9. The normalized transmissibility curves in Fig. 7-top show that the stop-band of the RDR was extended as requested. However, it is at the cost of slightly higher average values of 

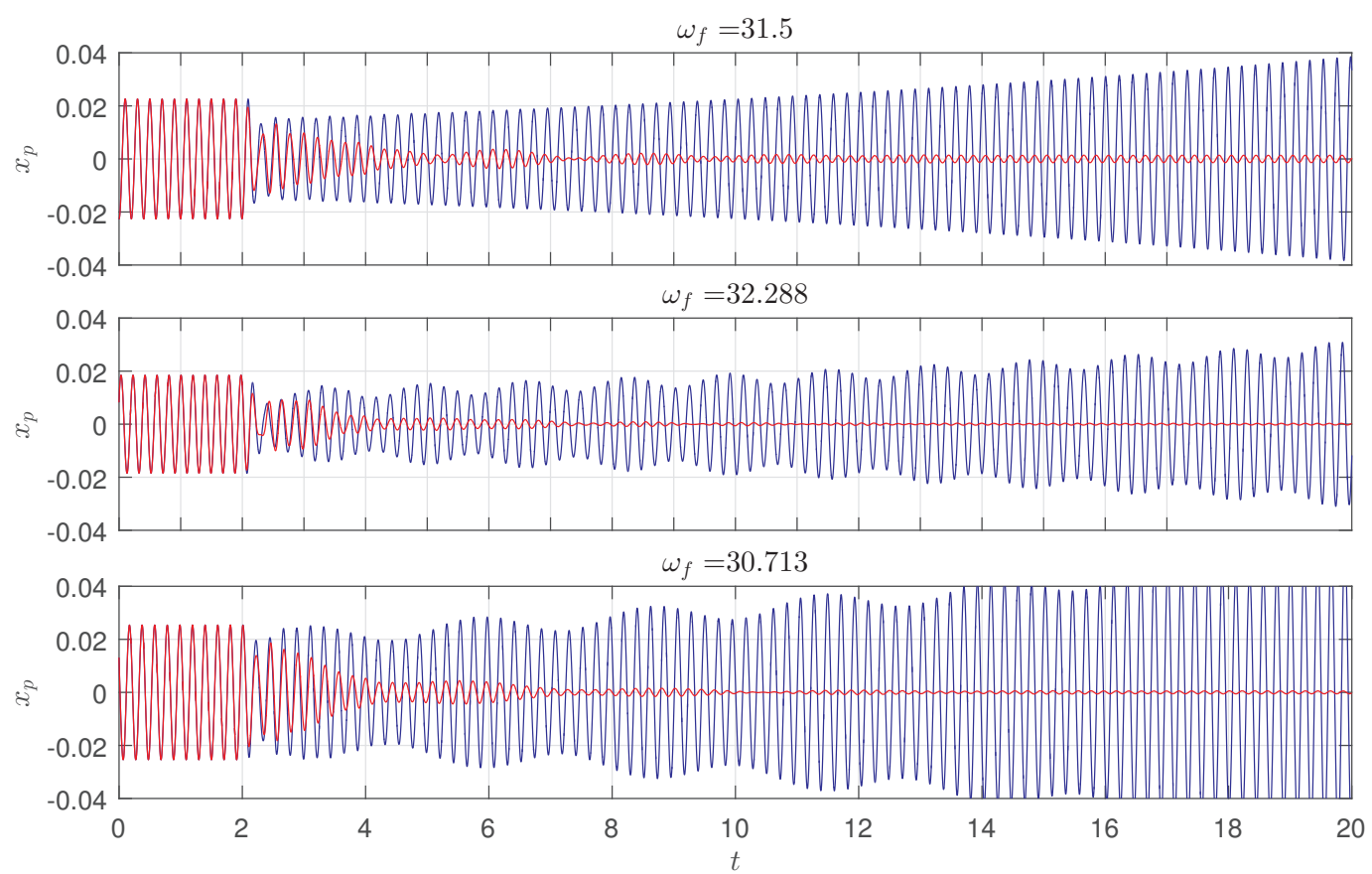

Fig. 9. Case 2 - Vibration suppression by DR (blue curve) and RDR (red curve) resonators, which are switched on at $t=2 \mathrm{~s}$, at three different frequencies. The nominal case with $\omega_{f_{n}}=31.5 \mathrm{~s}^{-1}$ is at the topmost figure.

$T(\omega)$ for $\omega \in I_{\omega}$ compared to Case 1 . The key benefit of the RDR can be seen from the spectral features depicted in Fig. 8. The overall set-up with the RDR is safely stable, satisfying the required spectral abscissa margin $\alpha<-0.5$. On the other, the set-up with the DR is unstable with a single couple of roots located slightly to the right of the stability boundary. The DR has the destabilizing effect on the overall structure, which is likely to be the case also for lower excitation frequencies. Recall that for the given damping $\zeta$ the DR becomes an unstable substructure for $\omega_{f}<0.78 \omega_{n}$ as discussed above based on [14]. Also in this case, the spectrum of the RDR has two dominant roots located close to the design excitation frequency root $j \omega_{f_{n}}$. In Fig. 9 with the simulations, it can be seen that the RDR performs well not only for the nominal frequency $\omega_{f_{n}}$, but also for the slightly modified frequencies by $\pm 2.5 \%$. On the other hand, in agreement with the spectral analysis, unstable responses are achieved for the set-up with DR. Thus, also this example confirms benefits of the proposed RDR concerning the stability and robustness imposed by the design. Let us remark that the stability with the classical DR could be recovered by applying a higher branch of the delay $\tau_{D R}$. For this purpose, the stability of the overall set-up with the DR can be analysed-analyzed by a CTCR method [16], leading to stabilizing gain and delay scheduling as recently applied in [38]. 


\section{Conclusions}

A new type of delayed resonator with a polynomial distribution of the delay is presented along with an optimization based design of its parameters. The first contribution is in achieving enhanced robustness of the resonator with respect to the mismatch between the design and true frequency of the vibration. The second contribution is in securing stability of the overall setup. In the design procedure, the normalized transmissibility characteristic of the resonator is turned to the objective function to be minimized over the proposed frequency stop-band centered at the nominal excitation frequency. Then, the interconnection of the resonator and the primary structure is formulated in a System of Delay Differential Algebraic Equations. The additional stability requirement is then given as a constraint. Applying the penalty method, the constrained optimization problem is turned to an unconstrained nonlinear optimization problem. The involvement of the spectral constraint in the objective function makes the optimization problem non-smooth, requiring targeted algorithms. The presented results show a successful implementation using HANSO [35]. The effectiveness of the proposed method is illustrated by means of two case study examples demonstrating an increased performance in comparison with the classical delayed resonator. Besides successful results are presented for the case where the classical approach would lead to an unstable system. The proposed resonator feedback design and its implementation are both considerably more complex compared to the design and implementation of the original delayed resonator with a lumped delay [5], [6]. Thus, it is suitable for applications where the frequency to be suppressed is not known accurately, i.e. it is not possible to tune the resonator ideally as assumed for the original delayed resonator. Note that by expressing the objective function in the optimization problem in terms of the averaged transmissibility function over a frequency range and by imposing a stability margin in the spectral abscissa constraint, the robustness of the vibration suppression against modeling mismatches and changes in system, resonator and environment is directly included in the resonator design. To see this, the vibration suppression is robustified against changes in the excitation frequency by favoring a flat behavior of the transmissibility function around the nominal frequency. But this flat behavior also implies robustness of the vibration suppression for a given excitation frequency against changes of the transmissibility function. Compared to a conventional design this reduces the chance that a re-tuning is necessary during the resonator's operation. However, if a re-tuning is needed, this is less intuitive due to more complicated structure of the feedback. An analysis of this trade-off is to be performed in the subsequent research, including experimental validation. 


\section{Acknowledgement}

The presented research has been supported by the Czech Science Foundation under the project 17-20943S. This work was also supported by the project C14/17/072 of the KU Leuven Research Council, by project G0A5317N of the Research Foundation-Flanders (FWO - Vlaanderen), and by the project UCoCoS, funded by the European Unions Horizon 2020 research and innovation programme under the Marie Sklodowska-Curie Grant Agreement No 675080.

\section{References}

[1] R. Rana, T. Soong, Parametric study and simplified design of tuned mass dampers, Engineering structures 20 (3) (1998) 193-204.

[2] M. B. Ozer, T. J. Royston, Extending den hartogs vibration absorber technique to multi-degree-of-freedom systems, Journal of Vibration and Acoustics 127 (4) (2005) 341-350.

[3] G.-L. Lin, C.-C. Lin, B.-C. Chen, T.-T. Soong, Vibration control performance of tuned mass dampers with resettable variable stiffness, Engineering Structures 83 (2015) 187-197.

[4] C. Verbaan, P. Rosielle, M. Steinbuch, Broadband damping of non-rigid-body resonances of planar positioning stages by tuned mass dampers, Mechatronics 24 (6) (2014) 712-723.

[5] N. Olgac, B. Holm-Hansen, A novel active vibration absorption technique: delayed resonator, Journal of Sound and Vibration 176 (1) (1994) 93-104.

[6] N. Olgac, Delayed resonators as active dynamic absorbers, US Patent 5,431,261 (Jul. 11 1995).

[7] D. Pilbauer, W. Michiels, T. Vyhlídal, A comparison of shaper-based and shaper-free architectures for feedforward compensation of flexible modes, TW Reports TW672.

[8] N. Olgac, Single mass dual frequency fixed delayed resonator, US Patent 5,505,282 (Apr. 9 1996).

[9] N. Olgac, M. Hosek, Active vibration absorption using delayed resonator with relative position measurement, Journal of vibration and acoustics 119 (1) (1997) $131-136$.

[10] M. Hosek, H. Elmali, N. Olgac, A tunable torsional vibration absorber: the centrifugal delayed resonator, Journal of Sound and Vibration 205 (2) (1997) $151-165$. 
[11] M. Hosek, N. Olgac, A single-step automatic tuning algorithm for the delayed resonator vibration absorber, Mechatronics, IEEE/ASME Transactions on 7 (2) (2002) 245-255.

[12] N. Jalili, N. Olgac, Multiple delayed resonator vibration absorbers for multidegree-of-freedom mechanical structures, Journal of Sound and Vibration 223 (4) (1999) 567-585.

[13] N. Alujević, I. Tomac, P. Gardonio, Tuneable vibration absorber using acceleration and displacement feedback, Journal of sound and vibration 331 (12) (2012) 2713-2728.

[14] T. Vyhlídal, N. Olgac, V. Kučera, Delayed resonator with acceleration feedback-complete stability analysis by spectral methods and vibration absorber design, Journal of Sound and Vibration 333 (25) (2014) 6781-6795.

[15] D. Pilbauer, T. Vyhlidal, N. Olgac, Delayed resonator with distributed delay in acceleration feedback - design and experimental verification, IEEE/ASME Transactions on Mechatronics 21 (4) (2016) 2120-2131.

[16] N. Olgac, R. Sipahi, The cluster treatment of characteristic roots and the neutral type time-delayed systems, Journal of dynamic systems, measurement, and control 127 (1) (2005) 88-97.

[17] V. Kucera, D. Pilbauer, T. Vyhlidal, N. Olgac, Extended delayed resonators design and experimental verification, Mechatronics 41 (2017) 29-41.

[18] H. Rivaz, R. Rohling, An active dynamic vibration absorber for a hand-held vibro-elastography probe, Journal of Vibration and Acoustics 129 (1) (2007) 101-112.

[19] J. G. Proakis, D. G. Manolakis, Digital signal processing: principles algorithms and applications (4th Edition), Prentice-Hall, Inc. Upper Saddle River, 2006.

[20] S. Vandewalle, W. Michiels, K. Verheyden, J. Vanbiervliet, A nonsmooth optimisation approach for the stabilisation of time-delay systems, ESAIM: Control, Optimisation and Calculus of Variations 14 (3) (2008) 478-493.

[21] R. Sipahi, S. Niculescu, C. Abdallah, W. Michiels, K. Gu, Stability and stabilization of systems with time delay, IEEE Control Systems Magazine 31 (1) (2011) 38-65.

[22] B.-L. Zhang, Q.-L. Han, X.-M. Zhang, X. Yu, Sliding mode control with mixed current and delayed states for offshore steel jacked platforms, Control Systems Technology, IEEE Transactions on 22 (5) (2014) 1769-1783.

[23] B.-L. Zhang, Q.-L. Han, X.-M. Zhang, Recent advances in vibration control of offshore platforms, Nonlinear Dynamics 89 (2) (2017) 755-771.

[24] A. Ramírez, R. Sipahi, S. Mondié, R. Garrido, An analytic approach to tuning of delay-based controllers for lti-siso systems, SIAM Journal on Control and Optimization 55 (1) (2017) 397-412. 
[25] E. Fridman, L. Shaikhet, Delay-induced stability of vector second-order systems via simple lyapunov functionals, Automatica 74 (2016) 288-296.

[26] M. E. Renzulli, R. Ghosh-Roy, N. Olgac, Robust control of the delayed resonator vibration absorber, Control Systems Technology, IEEE Transactions on 7 (6) (1999) 683-691.

[27] T. Vyhlídal, N. Olgac, V. Kučera, Design and stability analysis of delayed resonator with acceleration feedback, in: ASME 2013 Dynamic Systems and Control Conference, American Society of Mechanical Engineers, 2013, pp. V002T21A003-V002T21A003.

[28] T. Vyhlídal, M. Hromčík, Parameterization of input shapers with delays of various distribution, Automatica 59 (2015) 256-263.

[29] D. Pilbauer, W. Michiels, T. Vyhlídal, Distributed delay input shaper design by optimizing smooth kernel functions, Journal of the Franklin Institute 354 (2017) 5463-5485.

[30] J. Vaughan, A. Yano, W. Singhose, Comparison of robust input shapers, Journal of Sound and Vibration 315 (4) (2008) 797-815.

[31] W. Michiels, H. U. Ünal, Evaluating and approximating fir filters: an approach based on functions of matrices, IEEE Transactions on Automatic Control 60 (2) (2015) 463-468.

[32] W. Michiels, S.-I. Niculescu, Stability, Control, and Computation for TimeDelay Systems: An Eigenvalue-Based Approach, Vol. 27, SIAM, 2014.

[33] T. Vyhlídal, W. Michiels, P. McGahan, Synthesis of strongly stable statederivative controllers for a time-delay system using constrained non-smooth optimization, IMA Journal of Mathematical Control and Information 27 (2010) $437-455$.

[34] W. Michiels, Spectrum-based stability analysis and stabilisation of systems described by delay differential algebraic equations, IET control theory \& applications 5 (16) (2011) 1829-1842.

[35] M. Overton, HANSO: a hybrid algorithm for nonsmooth optimization, Available from cs. nyu. edu/overton/software/hanso.

[36] J. V. Burke, A. S. Lewis, M. L. Overton, A robust gradient sampling algorithm for nonsmooth, nonconvex optimization, SIAM Journal on Optimization 15 (3) (2005) 751-779.

[37] S. Mondié, W. Michiels, Finite spectrum assignment of unstable time-delay systems with a safe implementation, IEEE Transactions on Automatic Control 48 (12) (2003) 2207-2212.

[38] Q. Gao, A. S. Kammer, U. Zalluhoglu, N. Olgac, Combination of sign inverting and delay scheduling control concepts for multiple-delay dynamics, Systems \& Control Letters 77 (2015) 55-62. 